\title{
Pasti-Sorokin-Tonin actions in the presence of sources
}

\author{
R. Medina* and N. Berkovits ${ }^{\dagger}$ \\ Insituto de Física Teórica, Universidade Estadual Paulista, Rua Pamplona 145, 01405-900 São Paulo, Brazil
}

(Received 6 May 1997)

\begin{abstract}
Pasti, Sorokin, and Tonin have recently constructed manifestly Lorentz-invariant actions for self-dual field strengths and for Maxwell fields with manifest electromagnetic duality. Using the method of Deser et al., we generalize these actions in the presence of sources. [S0556-2821(97)04722-X]

PACS number(s): $04.40 . \mathrm{Nr}$
\end{abstract}

\section{INTRODUCTION}

Recent interest in duality has renewed the search for actions where duality symmetry and Lorentz invariance are manifest. At the present time, there are two types of actions where these symmetries are manifest. The first type of action is quadratic but contains an infinite number of fields. It was first discovered in two dimensions for describing chiral bosons [1], and later generalized to arbitrary self-dual fields [2] and to Maxwell fields with manifest electromagnetic duality [3]. In ten dimensions, it was rediscovered by analyzing the massless Ramond-Ramond sector of superstring field theory [4]. By studying the coupling of $D$-branes to the massless Ramond-Ramond string fields, this first type of action was generalized in the presence of sources [5].

The second type of manifestly covariant action with manifest duality contains a finite number of fields but is nonpolynomial in these fields $[6,7]$. It was discovered by Pasti, Sorokin, and Tonin (PST) and "covariantizes" actions found earlier $[8,9,10]$ which were manifestly dual but not manifestly Lorentz covariant. This PST action was inspired by an incorrect action of Khoudeir and Pantoja [11,12]. Equivalence among different formulations is of interest not only at a classical level [6], but also at a quantum level [13]. Recently, Deser et al. showed how to introduce sources into the nonmanifestly covariant versions of these actions [14]. Their method uses a field strength whose definition is modified in the presence of sources, as well as a coupling term of the type " $\vec{A} \cdot \vec{j}$. ."

In this paper, we "covariantize" their procedure, thereby generalizing the manifestly covariant PST actions in the presence of sources. In Sec. II of this paper, we generalize the PST action for self-dual fields [6], and in Sec. III, we generalize the PST action for Maxwell fields with manifest electromagnetic duality [7].

Our conventions are as follows: We use uncapitalized latin letters to denote space-time indices and capitalized latin letters to denote space indices. We work with the metric $g_{m n}=\operatorname{diag}(-1,+1, \ldots,+1)$. The antisymmetrization of space-time indices of a tensor is done without adding any additional factor, for example,

$$
B_{[l m] n}=B_{l m n}-B_{m l n} .
$$

\footnotetext{
*Electronic address: rmedina@power.ift.unesp.br

†Electronic address: nberkovi@ power.ift.unesp.br
}

\section{MANIFESTLY LORENTZ-INVARIANT CHIRAL 2-FORM ACTION WITH SOURCES}

In Ref. [6], Pasti, Sorokin, and Tonin write a manifestly Lorentz-invariant action for a chiral 2-form field, which is an antisymmetric field $A_{m n}(x)$ propagating in a $(5+1)$-dimensional Minkowski space-time whose field strength $F_{l m n}(x)$ is a self-dual field on shell. This means that

$$
F_{l m n}(x)=* F_{l m n}(x)
$$

where, in general, the dual of a field $C_{l m n}(x)$ is defined as

$$
* C_{l m n}(x)=\frac{1}{3 !} \epsilon_{l m n p q r} C^{p q r}(x)
$$

We are using the convention

$$
F_{l m n}(x)=\partial_{l} A_{m n}(x)+\partial_{m} A_{n l}(x)+\partial_{n} A_{l m}(x) .
$$

The generalization of the action of Ref. [6] in the presence of sources is

$$
\begin{aligned}
S= & \int d^{6} x\left(-\frac{1}{6} H_{l m n} H^{l m n}+\frac{1}{2\left(\partial_{q} a \partial^{q} a\right)}\right. \\
& \left.\times \partial^{m} a \mathcal{H}_{m n l} \mathcal{H}^{n l r} \partial_{r} a-A_{l n} j^{l n}\right),
\end{aligned}
$$

where $j^{l n}$ is the source (for self-dual fields the electric and magnetic sources are equal) and

$$
H_{l m n}=F_{l m n}+* G_{l m n}
$$

is the modified field strength. $F_{l m n}$ is still defined by Eq. (4) and satisfies Bianchi identities $\partial^{l}\left({ }^{*} F_{l m n}\right)=0 . G_{l m n}$ is defined to satisfy

$$
\partial_{l} G^{l m n}-j^{m n}=0 .
$$

$\mathcal{H}_{l m n}$ is an anti-self-dual field defined as

$$
\mathcal{H}_{l m n}=H_{l m n}-* H_{l m n} .
$$

The action in Eq. (5) is manifestly Lorentz invariant and coincides with the original action of Ref. [6] when there are no sources. We will show that it describes the dynamics of a 
chiral 2-form in the presence of sources by comparing this action, in a certain gauge, with the nonmanifestly covariant one of Ref. [14].

Making use of the identity $u^{[l} \mathcal{H}^{m n] s} u_{s}=2\left(u_{s} u^{s}\right) \mathcal{H}^{l m n}$ $+\epsilon^{l m n s u v} u^{w} u_{s} \mathcal{H}_{u v w}$ for $u_{l}=\partial_{l} a$, the equation of motion for $A_{m n}(x)$ leads to

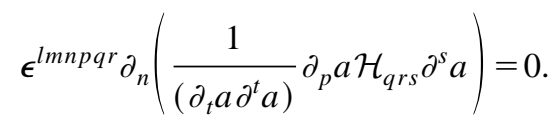

And now, considering the identity

$$
\epsilon^{l m n p q r} \partial_{n}\left(T_{l m} \partial_{p} a T_{q r}\right)=2 T_{l m} \epsilon^{l m n p q r} \partial_{n}\left(\partial_{p} a T_{q r}\right),
$$

in the case of $T_{l m}=\left[1 /\left(\partial_{k} a \partial^{k} a\right)\right] \mathcal{H}_{l m i} \partial^{i} a$, the equation of motion for $a(x)$ may be written as

$$
\begin{aligned}
& \left\{\frac{1}{\left(\partial_{k} a \partial^{k} a\right)} \mathcal{H}_{l m i} \partial^{i} a\right\} \\
& \quad \times\left\{\epsilon^{\text {lmnpqr }} \partial_{n}\left(\frac{1}{\left(\partial_{t} a \partial^{t} a\right)} \partial_{p} a \mathcal{H}_{q r s} \partial^{s} a\right)\right\}=0 .
\end{aligned}
$$

Thus, when considering Eq. (9), this last equation becomes simply an identity. This happens because $a(x)$ is a gauge field as can be seen by observing that the following gauge transformation leaves the action invariant:

$$
\delta a(x)=\phi(x), \quad \delta A_{m n}(x)=\frac{\phi(x)}{\left(\partial_{q} a \partial^{q} a\right)} \mathcal{H}_{m n s} \partial^{s} a .
$$

Since $a(x)$ transforms without derivatives, its gauge choice can be directly substituted in the action (5), leading to a Lorentz-noncovariant formulation of it. Choosing the gauge $\partial_{m} a=\delta_{m}^{0}$, this substitution leads to

$$
S=\int d^{6} x\left(-\frac{1}{6} H_{l m n} H^{l m n}+\frac{1}{2} \mathcal{H}_{0 n l} \mathcal{H}^{n l 0}-A_{l n} j^{l n}\right) .
$$

Using that $H_{l m n} H^{l m n}=3 H_{0 A B} H^{0 A B}-3 * H_{0 A B} * H^{0 A B}$ and identifying $E^{A B}=-H^{0 A B}$ and $B^{A B}=-* H^{0 A B}$ we end up with

$$
S=\int d^{6} x\left(E^{A B} B_{A B}-B^{A B} B_{A B}-A_{l n} j^{l n}\right),
$$

which, except for a factor $\frac{1}{4}$, is the action of Deser et al. [14].

\section{LORENTZ-COVARIANT FORMULATION FOR DUALITY-SYMMETRIC MAXWELL ACTION WITH SOURCES}

In this section we generalize the PST action for Maxwell fields [7] by considering the coupling of these fields to external sources. Their action is a Lorentz-invariant version of

\footnotetext{
${ }^{1}$ In deriving (13) we have made the following identification between the 5-index Levi-Cita symbol used in Ref. [14] and ours: $\epsilon^{A B C D E}=\epsilon^{A B C E D 0}$
}

the one found by Schwarz and Sen [10] in which, for the purpose of having manifest duality in the action, an additional Abelian field is included.

The procedure is straightforward from what we did in the previous section. In this case the action is

$$
\begin{aligned}
S= & \int d^{4} x\left[-\frac{1}{8} H_{m n}^{\alpha} H_{\alpha}^{m n}-\frac{1}{4\left(\partial_{q} a \partial^{q} a\right)} \partial^{m} a \mathcal{H}_{m n}^{\alpha} \mathcal{H}_{\alpha}^{n p} \partial_{p} a\right. \\
& \left.+\frac{1}{2} A_{m}^{\alpha} \epsilon_{\alpha \beta} j^{\beta m}\right],
\end{aligned}
$$

where the two Abelian fields are $A_{n}^{\alpha}(\alpha=1,2)$ and

$$
\begin{gathered}
F_{m n}^{\alpha}=\partial_{m} A_{n}^{\alpha}-\partial_{n} A_{m}^{\alpha}, \\
H_{m n}^{\alpha}=F_{m n}^{\alpha}+* G_{m n}^{\alpha}, \\
\mathcal{H}_{m n}^{\alpha}=\epsilon^{\alpha \beta} H_{\beta m n}-* H_{m n}^{\alpha} .
\end{gathered}
$$

$\epsilon^{\alpha \beta}$ is completely antisymmetric on its indices, with $\epsilon^{12}=1$. The metric tensor for the internal space is $g_{\alpha \beta}=\delta_{\alpha \beta}$.

Again, $G^{\alpha m n}$ is an external field, related to the sources by the condition

$$
\partial_{m} G^{\alpha m n}+j^{\alpha n}=0 .
$$

Our action (14) coincides with that of Pasti, Sorokin, and Tonin [7] when there are no sources. Note that the field $\mathcal{H}_{m n}^{\alpha}$ defined in Eq. (16) is self-dual with respect to the Lorentz and internal indices: $\mathcal{H}_{m n}^{\alpha}=\frac{1}{2} \epsilon^{\alpha \beta} \epsilon_{m n p q} \mathcal{H}_{\beta}^{p q}$.

Now, using the identity $u_{p} \mathcal{H}^{\alpha p n} u^{m}=-\left(u^{p} u_{p}\right) \mathcal{H}^{\alpha n m}$ $-\epsilon^{n m s t} \epsilon^{\alpha \beta} u^{q} \mathcal{H}_{\beta q s} u_{t}+u_{p} \mathcal{H}^{\alpha p m} u^{n}$ for $u_{l}=\partial_{l} a$, the equations of motion with respect to $A_{\alpha n}(x)$ and $a(x)$ lead, respectively, to

$$
\begin{gathered}
\epsilon^{m n p q} \partial_{n}\left[\frac{1}{\left(\partial_{s} a \partial^{s} a\right)} \partial_{p} a \mathcal{H}_{q r}^{\alpha} \partial^{r} a\right]=0, \\
\left\{\epsilon_{\beta \alpha} \frac{\partial^{l} a \mathcal{H}_{m l}^{\beta}}{\left(\partial_{k} a \partial^{k} a\right)}\right\}\left\{\epsilon^{m n p q} \partial_{n}\left[\frac{1}{\left(\partial_{s} a \partial^{s} a\right)} \partial_{p} a \mathcal{H}_{q r}^{\alpha} \partial^{r} a\right]\right\}=0 .
\end{gathered}
$$

Again, Eq. (20) becomes an identity when Eq. (19) is considered and this happens because $a(x)$ is a gauge field. This can be seen by noting that the following gauge transformation leaves the action (14) invariant:

$$
\delta a(x)=\phi(x), \quad \delta A_{m}^{\alpha}(x)=\phi(x) \frac{1}{\left(\partial_{q} a \partial^{q} a\right)} \epsilon^{\alpha \beta} \mathcal{H}_{\beta m n} \partial^{n} a
$$

In the same way as was noted in the previous section, as the transformation for $a(x)$ does not involve any derivatives, the gauge fixing of this field can be done at the level of the action. Using the gauge $\partial_{m} a=\delta_{m}^{0}$ and the identity

$$
H_{m n}^{\alpha} H_{\alpha}^{m n}=2 H_{0 N}^{\alpha} H_{\alpha}^{0 N}-2 * H_{0 N} * H_{0 N}(N=1,2,3),
$$


the action (14) adopts the form

$$
S=\frac{1}{2} \int d^{4} x\left[\epsilon_{\alpha \beta} \vec{B}^{\alpha} \cdot \vec{E}^{\prime \beta}-\vec{B}^{\alpha} \cdot \vec{B}_{\alpha}+A_{m}^{\alpha} \epsilon_{\alpha \beta} j^{\beta m}\right],
$$

where $E^{\prime \alpha N}=H^{\alpha 0 N}$ and $B^{\alpha N}=* H^{\alpha O N}$. In Eq. (22) we have $E^{\prime \alpha N}=E^{\alpha N}-\partial^{N} A^{\alpha 0}$, where $E^{\alpha N}$ is the "electric field" appearing in [14]. The action (22) coincides with the Maxwell action of Ref. [14], in the case of external sources, after canceling the term $A_{0}^{\alpha} \epsilon_{\alpha \beta} j^{\beta 0}$ with the contribution coming from $\partial^{N} A^{\alpha 0}$ in $E^{\prime \alpha N}$.

\section{ACKNOWLEDGMENTS}

R.M. ackowledges support from FAPESP Grant No. 96/ 00702-8. N.B. would like to acknowledge the financial support of FAPESP Grant No. 96/05524-0 and useful conversations with S. Deser and D. Sorokin.
[1] B. McClain, Y. S. Wu, and F. Yu, Nucl. Phys. B343, 689 (1990); C. Wotzasek, Phys. Rev. Lett. 66, 129 (1991).

[2] F. P. Devecchi and M. Henneaux, Phys. Rev. D 54, 1606 (1996); I. Bengtsson and A. Kleppe, Int. J. Mod. Phys. A 12, 3397 (1997).

[3] I. Martin and A. Restuccia, Phys. Lett. B 323, 311 (1994).

[4] N. Berkovits, Phys. Lett. B 388, 743 (1996).

[5] N. Berkovits, Phys. Lett. B 395, 28 (1997).

[6] P. Pasti, D. Sorokin, and M. Tonin, Phys. Rev. D 55, 6292 (1997).

[7] P. Pasti, D. Sorokin, and M. Tonin, Phys. Rev. D 52, 4277 (1995).
[8] D. Zwanziger, Phys. Rev. D 3, 880 (1971).

[9] S. Deser and C. Teitelboim, Phys. Rev. D 13, 1592 (1976).

[10] J. H. Schwarz and A. Sen, Nucl. Phys. B411, 35 (1994).

[11] A. Khoudeir and N. Pantoja, Phys. Rev. D 53, 5974 (1996).

[12] P. Pasti, D. Sorokin, and M. Tonin, Phys. Rev. D 56, 2473 (1997).

[13] H. O. Girotti, M. Gomes, V. O. Rivelles, and A. J. da Silva, this issue, Phys. Rev. D 56, 6615 (1997).

[14] S. Deser, A. Gomberoff, M. Henneaux, and C. Teitelboim, Phys. Lett. B 400, 80 (1997). 